\title{
On Some Infinite Series Related to the Twin Primes
}

\author{
A. Dinculescu*
}

\author{
“Esperion” at Western Michigan University, 5110 McCracken Hall, Kalamazoo, MI 49008, USA
}

\begin{abstract}
It is shown that all positive integers can be divided into numbers that can lead to a pair of twin primes by a simple algebraic operation and numbers that cannot. The paper devises a formula for finding the numbers in each category, and shows that the numbers in the second category can be arranged in infinite groups and super-groups with an inner symmetry, a precise interval length and a well-defined number of terms. After presenting some of their properties, the paper analyzes a relatively small interval remained after subtracting certain numbers that belong to the second category from the set of positive integers in the interval. Because all terms in the interval depend on a single prime $P$ to fall into one category or another, the fraction of terms from the second category is about $2 / P$ of the total number of terms. The rest must belong to the first category.
\end{abstract}

Keywords: (2010 MSC): primes (11A41), distribution of primes (11N05), sieves (11N35).

\section{INTRODUCTION}

According to the Twin Prime Conjecture [1] there are an infinite number of pairs of twin primes. This conjecture has not been proven yet, the main difficulty being the fact that probabilistic events for consecutive primes are not truly independent [2]. Here we present an indirect approach to this problem characterized by the fact that, instead of looking at the chance of having twin primes, it looks at the conditions that must be satisfied for not having them. For that, we take a closer look at those numbers that cannot lead to a pair of twin primes by a simple algebraic operation and at the way they are generated, and try to determine if, starting from a certain number, all positive integers can be formed only from this type of numbers. Although this approach involves some simple approximations, it does not require probabilistic estimates.

\section{OBJECTIVE OF THE WORK}

1. Simplify the problem by dividing the set of positive integers into two and only two categories: numbers that can lead to a pair of twin primes by the same algebraic operation, and numbers that cannot.

2. Devise a formula for calculating the numbers that belong to the second category in a given interval.

3. Show that these numbers display an inner symmetry and obey certain rules that might shed light on the numbers in the first category.

4. Based on the characteristics of the numbers in the second category, find an approximate formula for calculating the numbers that belong to the first category in a given interval.

*Address correspondence to this author at the 4148 NW 34th Drive, Gainesville, Florida, 32605, USA; E-mail: adinculescu@cox.net Tel: 352-337-2966

\section{TWIN RANKS AND NON-RANKS}

One can associate to each pair of twin primes $P$ and $P+2$ a twin index $K=P+1$ representing the number between them. Since all primes $P$ with the exception of 2 and 3 are of the form $P= \pm 1(\bmod 6)$, all twin indices except 4 are of the form $K=6 n$, where $n$ is a positive integer. This allows one to define a "twin rank" as $k_{*}=K / 6$. The number $K^{2}-1=P(P+2)$ is divisible only by $P$ and $P+2$. Therefore, if a number $(6 n)^{2}-1=(6 n+1)(6 n-1)$ is not divisible by any prime $P \leq \sqrt{6 n+1}$, then $n=k_{*}$ is a twin rank. As can be easily seen, all twin ranks lead to a pair of twin primes $6 k_{*}+1$ and $6 k_{*}-1$ by the same algebraic operation. Conversely, any number $k$ that satisfies

$k=n P \pm[P / 6]$

is a "non-rank". Non-ranks cannot lead to a pair of twin primes by the same algebraic operation. (Here $[x]$ means the nearest integer to $x$ ).

It is easy to see that $[P / 6]$ is actually the integer obtained by dividing either $\mathrm{E}^{P}+1$ er $\mathrm{P}-1$ by 6 . With eq. (1) one can find all non-ranks smaller than

by using all primes $M_{j+1}=\left(P_{j+1}^{2}-1\right) / 6$ By subtracting these non-rapiks from the set of positive integers $n \leq\left(P_{j+1}^{2}-1\right) / 6$ one obtains all twin ranks and, hence, all twin primes with indices $k_{*}<\left(P_{j+1}^{2}-1\right) / 6$ $K<\left(P_{j+1}^{2}-1\right)$. We used the sign $"<$ instead of $" \leq$ in the last two expressions because a number of the form $P^{2}-1$ 
cannot be a twin index and, consequently, a number of the form $\left(P^{2}-1\right) / 6$ cannot be a twin rank.

One might wonder if the set of positive integers contains other numbers beside twin ranks and non-ranks. Here is a simple way of showing that there are no other numbers in the set of positive integers beside twin ranks and non-ranks:

a) Take all natural numbers $n$ up to a certain number $N$ and put them on a line; call it " the line $n$ ";

b) Multiply the numbers on this line by 6 and put them on a parallel line; call it "the line $6 n$ "; obviously there is a one to one correspondence between the numbers on the line $n$ and the numbers on the line $6 n$;

c) For each $6 n$ divide $6 n+1$ and $6 n-1$ by $P$ up to $P \leq \sqrt{6 n+1}$;

d) Define non-ranks as all $n$ for which $(6 n+1) / P$ or $(6 n-1) / P$ are integers and cross them out on the line $n$; do the same with their correspondents on the line $6 n$;

e) What remain uncrossed on line $6 n$ are all numbers for which neither $(6 n+1) / P$ nor $(6 n-1) / P$ are integers; as shown, these are twin indices $K$;

f) By definition, their correspondents $k_{*}=K / 6$ on line $n$ are twin ranks; obviously, there are no other numbers left on this line beside non-ranks and twinranks;

g) Since 1, 2 and 3 on the line $n$ are twin ranks, what remain on the line $n$ after subtracting the non-ranks from the set of positive integers are only twin ranks.

It is important to realize that while many of the non-ranks $k_{j}$ determined with eq. (1) using a prime $P_{j}$ can be found with the help of primes smaller than $P_{j}$ none of them can be found using primes larger than $P_{j}$. This property is very important because it ensures that once a number was shown to be a twin rank by all primes up to a certain prime, it is not going to be "covered" (i.e. shown to be a non-rank) by larger primes. In the following paragraphs we present a series of propositions underlining the properties of non-ranks that might shed light on the characteristics of twin ranks.

\section{GROUPS, SUPER-GROUPS AND REMNANTS}

From eq. (1) it is easy to see that:

Proposition no. 1: All non-ranks are symmetrically distributed at equal distances $[P / 6]$ from the numbers that are multiple of a prime $P$.

There are, therefore, on the line of natural numbers, two non-ranks associated to each prime $P$ in each interval of length $\Delta n=P$ starting with $[P / 6]$. Because a number can be multiple of several primes, we grouped the non-ranks by their "parent prime" defined as the smallest prime required by (1) to find them.

Example 1: Here are the first 10 non-ranks of parent prime $P_{17}=59: 777,1013,1052,1288,1347,1603,1642$, 1760, 1937, 1957.

All of the above numbers are of the form $k_{17}=59 n \pm[59 / 6]$, where $[59 / 6]=10$ is the nearest integer to $[59 / 6]$. Although writing a recurrence formula for non-ranks was not one of the objective of this paper, we would like to point out that all non-ranks of parent prime 5 end in 1, 4, 6 or 9 because all multiples of 5 end in 0 or 5 . This leads to the conclusion that none of the twin ranks end in $1,4,6$ or 9. This characteristic and the fact that the reminder from the division of a twin rank $k_{*}$ by a prime $P<k_{*}$ cannot take the values $[P / 6]$ or $P-[P / 6]$ (see eq. (1) are important properties of twin ranks, and allow one to write the following recurrence formulae for the non-ranks $k_{3}$ and $k_{4}$ of parent primes $P_{3}=5$ and $P_{4}=7$, respectively. One has:

$k_{3}=k_{3 a} \cup k_{3 b}$ with $k_{3 a}=5 n-1$ and $k_{3 b}=k_{3 a}+2$.

One also has: $k_{4}=k_{4 a i} \cup k_{4 b}$ with $k_{4 a 1}=7(10 n-8)-1$,

$k_{4 a 2}=7(10 n-7)-1, k_{4 a 3}=7(10 n-6)-1$,

$k_{4 a 4}=7(10 n-3)-1, k_{4 a 5}=7(10 n-2)-1$,

$k_{4 a 6}=7(10 n-1)-1$, and $k_{4 b}=k_{4 a i}-5$, where $n=1,2,3$.

Note that together these two categories of non-ranks represent more than $57 \%$ of all existing non-ranks.

Since the non-ranks with different parent primes are mixed together on the line of positive integers, one expects to see a pattern in their distribution in an interval on the order of the least common multiple of all corresponding primes up to their largest parent prime. This property together with other characteristics of the non-ranks is listed below.

Proposition no. 2: The non-ranks $k_{j}$ of parent prime $P_{j}$ form an infinite number of consecutive groups "of order" ${ }^{j}$ of equal interval lengths $L_{j}$ each of them containing $G_{j}$ non-ranks, with the same gap between "equivalent terms", i.e. between the non-ranks that occupy the same positions in a group as in the first group.

Once a term in the first group is known, one can find the equivalent terms in all the other groups of the same parent prime by simply adding to that term the length $L_{j}$ multiplied by an integer. One has $k_{x n j}=k_{x 1 j}+n L_{j}$, where $n=0,1,2, .$. and the index $x n j$ means the $x^{\text {th }}$ term in the $n^{\text {th }}$ group of order $j$. This equation allows one to write another recurrence formula for the non-ranks $k_{4}$. One has: 


$$
\begin{aligned}
k_{4}= & (8+35 n) \cup(13+35 n) \cup(15+35 n) \cup(20+35 n) \\
& \bigcup(22+35 n) \cup(27+35 n)
\end{aligned}
$$

where $8,13,15,20,22,27$ are the non-ranks of parent prime $P_{4}=7$ in the first group of order $j=4$ and $n=0,1,2$..

Proposition no. 3: Each group of order $\mathrm{j}$ has a central gap between the pair of consecutive terms whose sum equals $L_{j}$. Except for the groups of the first 2 orders, the central gap occurs after $G_{j} / 2$ terms.

Proposition no. 4: The gaps between consecutive terms in a group are symmetrically distributed on each side of the central gap. As a consequence, the fractions of non-ranks in two intervals of equal length, situated at equal distances from the central gap, are equal.

Proposition no. 5: The sum of the terms situated at an equal distance from the central gap in a group is constant and equal to $L_{j}$.

Proposition no. 6: Except for the groups of the first 2 orders, the sum of all terms in a group of order $\mathrm{j}$ is $\sum_{k_{i} \in G_{j}} k_{i}=L_{j} G_{j} / 2$

Proposition no. 7: The interval length of a group of order $\mathrm{j}$ is the least common multiple of all intervals $\Delta n=P_{i}$, where $5 \leq P_{i} \leq P_{j}$. One has $L_{j}=\prod_{i=3}^{j} P_{i}$.

In order to find the number $G_{j}$ of non-ranks in a group of order $j$ one has to eliminate all non-ranks related to primes smaller than $j$. One starts by subtracting a fraction $2 / 5$ from the interval $L_{j}$. From the remaining interval one subtracts a fraction of $2 / 7$ and so on up to $2 / P_{j-1}$ when one obtains $\prod_{i=3}^{j-1}\left(P_{i}-2\right)$. The non-ranks $k_{j}$ that have not been covered by any prime smaller than $P_{j}$ represent a fraction $2 / P_{j}$ of this number, hence:

Proposition no. 8: The number of non-ranks in a group of order $j$ is $G_{j}=2 \prod_{i=3}^{j-1}\left(P_{i}-2\right)$. Note that because $L_{j}=\prod_{i=3}^{j} P_{i}$ the fractions obtained in the above computations are not approximate numbers but exact integers.

Proposition no. 9: The fraction of non-ranks of parent prime $P_{j}$ in the interval occupied by a group of order $\mathrm{j}$ is

$q_{j}=\frac{G_{j}}{L_{j}}=\frac{2}{P_{j}} \prod_{i=3}^{j} \frac{P_{i}-2}{P_{i}}$
Because one has to use all primes up to a certain prime $P_{j} \quad$ in order to find the non-ranks smaller $M_{j+1}=\left(P_{j+1}^{2}-1\right) / 6$, one needs to know all non-ranks of parent primes $5 \leq P_{i} \leq P_{j}$. The totality of these non-ranks form a super-group of order $j$.

Proposition no. 10: The non-ranks of parent primes $5 \leq P_{i} \leq P_{j}$ form an infinite number of consecutive supergroups of order $\mathrm{j}$ of equal interval lengths $L_{j}$ each of them containing $S_{j}$ terms, with the same gap between equivalent terms, i.e. between the non-ranks that occupy the same positions in a super-group as in the first super-group.

Example 2: Here are the first 62 non-ranks of parent primes $5 \leq P_{i} \leq P_{j}$ in the super-group $S_{7}: 4,6,8,9,11,13$, $14,15,16,19,20,21,22,24,26,27,28,29,31,34,35,36$, $37,39,41,42,43,44,46,48,49,50,51,53,54,55,56,57$, $59,61,62,63,64,65,66,67,68,69,71,74,75,76,78,79$, $80,81,82,83,84,85,86,88$.

The length of a super-group equals the length of the group of the largest parent prime in the super-group. (Note that, due to the fact that 5 is the smallest prime that can be used in eq. (1), all first super-groups begin at $1+3=4$ and end at $L+3)$. As before, once a term in the first super-group is known, one can find the equivalent terms in all the other super-groups of the same parent prime by simply adding to that term $L_{j}$ multiplied by an integer. One has $k_{x n j}=k_{x 1 j}+n L_{j}$, where $n=0,1,2, .$.

Proposition no. 11: Each super-group of order $j$ has a central gap $g_{s}$ between the terms $S_{c 1}=\left(L_{j}-3\right) / 2$ and $S_{c 2}=\left(L_{j}+3\right) / 2$. Since $6 S_{c 1}-1=5\left(3 L_{j} / 5-2\right) \quad$ and $6 S_{c 2}+1=5\left(3 L_{j} / 5+2\right)$ are composite numbers, $S_{c 1}$ and $S_{c 2}$ are consecutive non-ranks and the central gap in all super-groups has the same value $g_{s}=3$.

Proposition no. 12: The gaps between consecutive terms in a super-group are symmetrically distributed on each side of the central gap. As a consequence, the fractions of nonranks in two intervals of equal length, situated at equal distances from the central gap, are equal.

Proposition no. 13: The sum of the terms situated at an equal distance from the central gap in a super-group of order $\mathrm{j}$ is constant and equal to $L_{j}$.

Proposition no. 14: Except for the super-groups of the first 3 orders the sum of all terms in a super-group of order $j$ is $\sum_{k_{i} \in S_{j}} k_{i}=L_{j}\left(S_{j} / 2+3\right)$. This sum is also given by the following iteration formula valid for all super-groups: 


$$
\sum_{k_{i} \in S_{j}} k_{i}=\sum_{k_{i} \in S_{j-1}} k_{i}+L_{j-1} S_{j-1} \frac{P_{j}\left(P_{j}-1\right)}{2}+\sum_{k_{i} \in G_{j}} k_{i}
$$

Proposition no. 15: Each super-group of order $\mathrm{j}$ contains an integer number of nested groups of parent primes $5 \leq P_{i} \leq P_{j}$. This number is given by the ratio of the length of the super-group of order $j$ to the length of the group of order i. Accordingly,

Proposition no. 16: The number of terms of parent prime $P_{i}$ in a super-group of order $j>i$ is

$$
G_{i j}=\frac{L_{j}}{L_{i}} G_{i}=\frac{2 L_{j}}{P_{i}} \prod_{a=3}^{i-1} \frac{P_{a}-2}{P_{a}}=q_{i} L_{j}
$$

It follows that, the fraction of non-ranks of parent prime $P_{i}$ in the interval occupied by a super-group of order $j$ is the same as their fraction in the interval occupied by the group of order $i$ :

$$
q_{i}=\frac{G_{i j}}{L_{j}}=\frac{G_{i}}{L_{i}}=\frac{2}{P_{i}} \prod_{a=3}^{i-1} \frac{P_{a}-2}{P_{a}}
$$

Proposition no. 17: The number of non-ranks in a supergroup is the sum of the number of non-ranks in the constituent groups:

$$
S_{j}=L_{j} \sum_{i=3}^{j} q_{i}=L_{j}\left(1-\prod_{i=3}^{j} \frac{P_{i}-2}{P_{i}}\right)
$$

Proposition no. 18: The fraction of all non-ranks in the interval occupied by a super-group of order $j$ is

$$
Q_{j}=\frac{S_{j}}{L_{j}}=\sum_{i=3}^{j} q_{i}=1-\prod_{i=3}^{j} \frac{P_{i}-2}{P_{i}}
$$

Since $L_{j}>S_{j}$ there will always be "remnants" in $L_{j}$ not included in $S_{j}$. They consist of twin-ranks and non-ranks of parent primes larger than $P_{j}$. As can easily be seen:

Proposition no. 19: The number $R_{j}$ of terms in a remnant from a super-group of order $\mathrm{j}$ is half the number of terms in the next group:

$$
R_{j}=L_{j}-S_{j}=L_{j}\left(1-Q_{j}\right)=\prod_{i=3}^{j}\left(P_{i}-2\right)=\frac{1}{2} G_{j+1}
$$

Proposition no. 20: The fraction of terms belonging to a remnant from a super-group of order $j$ in the corresponding interval is

$x_{j}=\frac{R_{j}}{L_{j}}=1-Q_{j}=\prod_{i=3}^{j} \frac{P_{i}-2}{P_{i}}$

Proposition no. 21: The sum of all terms in a remnant is given by the difference between the sum of all numbers in the interval occupied by the super-group and the sum of the terms in that super-group:

$$
\sum_{k_{i} \in R_{i}} k_{i}=\frac{\left(L_{j}+3\right)\left(L_{j}+4\right)}{2}-\sum_{k_{i} \in S_{i}} k_{i}-6
$$

(For a better understanding of this equation, recall that all first super-groups begin at $1+3=4$ and end at $S_{j}+3$ ). With $\sum_{k_{i} \in S_{j}} k_{i}$ a multiple of $L_{j}$ and $(3 \times 4) / 2-6=0$, it is easy to see that:

Proposition no. 22: The sums of all terms in a group, a super-group or a remnant are multiple of their interval lengths:

$$
\sum_{k_{i} \in G, S, R} k_{i}=n L
$$

As shown, the twin ranks that can be found with eq. (1) using all primes up to $P_{j}$ cannot be larger than $M_{j+1}=\left(P_{j+1}^{2}-1\right) / 6$. Therefore:

Proposition no. 23: All terms smaller than $M_{j+1}$ in a remnant from a super-group of order $j$ are twin ranks. (We call these numbers "front twin ranks", and the interval $1 \ldots M_{j+1}$ "the first interval").

Example 3: The following are the front twin ranks that remain after subtracting the non-ranks of parent primes $5 \leq P \leq 17$ given in Example 2 from the first natural numbers up to $\left(19^{2}-1\right) / 6=60: 1,2,3,5,7,10,12,17,18$, $23,25,30,32,33,38,40,45,47,52,58$. When multiplied by 6 and added \pm 1 these twin ranks give all twin primes smaller than 360 .

Proposition no. 24: Starting with $P_{5}=11$ all remnant have a central gap $g_{R}=1$ between the numbers $\left(L_{j}-1\right) / 2$ and $\left(L_{j}+1\right) / 2$.

Proposition no. 25: The gaps between consecutive terms in a remnant are symmetrically distributed on each side of the central gap.

Proposition no. 26: The sum of the terms situated at an equal distance from the central gap in a remnant from a super-group of order $\mathrm{j}$ is constant and equal to $L_{j}$.

Before going to the next section, we would like to draw the reader's attention to the fact that all quantities associated so far with groups, super-groups and remnants were deterministic and not probabilistic. Unlike the estimation of the number of twin primes in a given interval, here one can say exactly how many non-ranks are in a group or a super-group, and how many terms are in a remnant, regardless of their sizes. 


\section{TOO MANY NUMBERS, TOO FEW PRIMES}

Let us see now if it is possible to have only non-ranks in a remnant $R_{j}$ beyond the first interval. For a negative answer it is enough to look only at a relatively small interval ("the second interval") after the front twin ranks. This interval is located between $M_{j+1}$ were the front twin ranks end, and $M_{j+2}$ where the use of primes larger than $P_{j+1}$ for finding non-ranks become necessary. It has a length $\Delta M_{j+1}=\left(P_{j+2}^{2}-P_{j+1}^{2}\right) / 6$ and contains $\Lambda_{j}$ terms, all of them twin ranks or non-ranks of the same parent prime $P_{j+1}$. A minimum requirement for having only non-ranks in a remnant $R_{j}$ (except for the front twin ranks) is that all terms in the second interval $\Lambda_{j}$ be covered by the prime $P_{j+1}$. As shown, the numbers of the form $\left(P^{2}-1\right) / 6$ are non-ranks, hence the first possible number $M_{j+1}$ in the second interval is a non-rank, but it does not have to be of parent prime $P_{j+1}$. Sometimes it is, sometimes it is not. When it is not of parent prime $P_{j+1}$ the number $M_{j+1}$ is not part of $\Lambda_{j}$. In this case the smallest non-rank of parent prime $P_{j+1}$ in the interval is $k_{\lambda 1}>M_{j+1}$. Obviously, there can't be any nonranks in $\Lambda_{j}$ with a value between $M_{j+1}$ and $k_{\lambda 1}$ because all non-ranks of parent primes smaller or equal to $P_{j}$ are in $S_{j}$ and the non-ranks of larger parent primes are beyond $k_{\lambda 1}$. Therefore:

Proposition no. 27: When the first non-rank in the second interval is different from $M_{j+1}$ all remnants with values between $M_{j+1}$ and $k_{\lambda 1}$ are twin ranks.

Since the gaps between consecutive terms in a remnant are symmetrically distributed on each side of the central gap, the fraction of remnants in two intervals of equal length situated at equal distances from the central gap are equal. This means that the fraction of remnants at the beginning (where the second interval is located) does not differ too much from the fraction at the end, and both of them cannot be too different from the average value $x_{j}=1-Q_{j}$ given by eq. (3). Accordingly, we approximate the number of remnants in the second interval as a fraction $x_{j}$ of the length $\Delta M_{j+1}$ of the interval. One has

$\Lambda_{j} \cong x_{j} \Delta M_{j+1}=\Delta M_{j+1} \prod_{i=3}^{j} \frac{P_{i}-2}{P_{i}}$

Although fairly accurate, this is not a precise value. However, what matters here is not the exact number of terms in the interval, but the fraction of them that can be covered by a single prime. In this case the prime is $P_{j+1}$ because the non-ranks of smaller parent primes are not in $R_{j}$, and primes larger than $P_{j+1}$ cannot cover numbers smaller than $M_{j+2}$. From eq. (2) we know that the fraction of non-ranks of parent prime $P_{j+1}$ in a group of order $j$ is $q_{i}$. Therefore, based on the same reasoning as before applied this time to a group, we approximate the number $r_{j}$ of non-ranks of parent prime $P_{j+1}$ in the second interval as a fraction $q_{j+1}$ of the length of the interval. One has:

$$
r_{j} \cong q_{j+1} \Delta M_{j+1}=\frac{2 \Delta M_{j+1}}{P_{j+1}} \prod_{i=3}^{j} \frac{P_{i}-2}{P_{i}}=\frac{2}{P_{j+1}} \Lambda_{j}
$$

This again is not a precise value, but the error is so small compared to the factor $P_{j+1} / 2$ needed to cover all terms in $\Lambda_{j}$ that it does not affect in any way the final result. Hence:

Proposition no. 28: A prime $P_{j+1}$ can cover only a fraction of about $2 / P_{j+1}$ of the number $\Lambda_{j}$ of terms in the second interval of a remnant from a super-group of order $j$. The rest are twin ranks. Since there are infinitely many super-groups each of them with a remnant and a first interval, one cannot escape the (heuristic) conclusion that the existence of twin ranks cannot be avoided and, therefore, there are infinitely many twin primes.

Example 4: The following numbers: $60,70,72,73,77$, 87 are the terms in the second interval $\Lambda_{7}$ belonging to the remnant $R_{7}$. They were obtained by subtracting the corresponding non-ranks of parent primes $5 \leq P \leq 17$ in the super-group $S_{7}$ (given in Example 2) from the interval of positive integers $M_{8} . . M_{9}=60 . .88$. As can easily be seen, the only non-ranks in this interval are $3 P_{8}+3=60$ and $4 P_{8}-3=73$, both of parent prime $P_{8}=19$. The remaining numbers: 70, 72, 77, 87 are twin ranks. To them correspond the following twin primes: 419, 421, 431, 433, 461, 463, 521 , and 523.

Now, a very important question arises: Is it possible that, starting from a certain number $\mathrm{N}$ to have all integers larger than $\mathrm{N}$ covered by primes $P \leq \sqrt{6 \mathrm{~N}+1}$ ? Based on Propositions 10, 12 and 19, the answer is "no". If that were the case, then:

a) The gaps at the beginning of a super-group (where the front twin ranks are) would be different from the gaps at the beginning of the other super-groups of the same order (were in the absence of twin ranks and nonranks of larger parent prime all gaps would be equal to 1 );

b) There would be no symmetry between the gaps at the beginning of a super-group and the gaps at the end as shown in Proposition 12; and 
c) The number of remnants from a super-groups would be much smaller than that given by the Proposition 19.

Also, it can be argued that since $1-Q_{j}$ approaches zero as $Q_{j}$ approaches 1 , in the long run there will be no terms in the second interval, and hence no twin ranks. That this is not the case can be seen from the following

Lemma: The number of terms in the second interval is larger than the gap between the primes that determine the length of the interval.

Proof: For any prime $P_{i}$ one has $P_{i}-2 \geq P_{i-1}$, hence by eq. (4) one has

$\Lambda_{j} \geq \frac{3}{P_{j}} \Delta M_{j} \geq \frac{\left(P_{j+2}+P_{j+1}\right)\left(P_{j+2}-P_{j+1}\right)}{2 P_{j}}$

With $P_{j+2}+P_{j+1}>2 P_{j} \quad$ one has $\Lambda_{j}>P_{j+2}-P_{j+1}$. This completes the proof. Based on this result we are asserting the following

Conjecture: Given two consecutive primes $P_{j+1}$ and $P_{j+2}$, there exists on average at least one pair of twin primes in the interval $P_{j+1}^{2} \ldots P_{j+2}^{2}$

Tentative proof: According to The Prime Number Theorem [3], the "average length" of the gap between a prime $P$ and the next prime is of the order of $\ln P$. Hence, by eq. (5) the number of terms in the second interval becomes larger and larger as one goes up on the line of natural numbers, and so does the number of terms in the second interval that cannot be covered by a single prime.

\section{APPROXIMATE FORMULA FOR THE NUMBER OF TWIN RANKS}

Since $x_{j}$ is a characteristic of the whole super-group, eq. (4) is not specific to the second interval. In principle one can apply it to any interval $\Delta M_{i}$ inside a remnant $R_{j}$ if one wants to approximate the number of terms (twin ranks and non-ranks) in that interval. A special case however is the first interval. In this case, because the interval contains only twin ranks, eq. (4) represents an estimate of the number $F_{T j}$ of the front twin ranks and, hence, of the number of twin primes up to $6 M_{j+1}$.

From $C_{2}=\prod_{i=2}^{\infty} \frac{P_{i}\left(P_{i}-2\right)}{\left(P_{i}-1\right)^{2}}$, where $C_{2}=0.66 \ldots$ is the twin prime constant [4], and $\prod_{i=1}^{j} \frac{P_{i}-1}{P_{i}} \approx \frac{e^{-\gamma}}{\ln P_{j}}$, where $\gamma=0.57721 \ldots$ is Euler's constant [5], one obtains $x_{j}=\prod_{i=3}^{j} \frac{P_{i}-2}{P_{i}} \approx 3 C_{2}\left(\frac{2 e^{-\gamma}}{\ln P_{j}}\right)^{2}$. Equation (4) applied to the

first interval then reads

$$
F_{T j} \approx 3 C_{2}\left(\frac{2 e^{-\gamma}}{\ln P_{j}}\right)^{2} M_{j} \approx \frac{2 C_{2}}{e^{2 \gamma}} \frac{P_{j}^{2}}{\ln ^{2} P_{j}}
$$

Now, if one wants to approximate the number $L_{T j}$ of twin ranks in the entire interval $L_{j}=\prod_{i=3}^{j} P_{i}$ occupied by a super-group of order $j$, one can consider $L_{j}$ as the first interval $M_{z}$ of a much larger super-group of order $z$. In this case $\quad L_{j} \approx M_{z} \approx P_{z}^{2} / 6 . \quad$ With $\quad 6 L_{j}=\prod_{i=1}^{j} P_{i}$,

$\ln \prod_{i=1}^{j} P_{i}=\sum_{i=1}^{j} \ln P_{i} \approx P_{j}$ and $P_{j}^{2} \cong 6 M_{j}$ eq. (6) gives

$L_{T j} \approx 3 C_{2}\left(\frac{2 e^{-\gamma}}{\ln P_{z}}\right) M_{z} \approx \frac{12 C_{2}}{e^{2 \gamma}} \frac{L_{j}}{\ln ^{2}\left(6 L_{j}\right)} \approx \frac{12 C_{2}}{e^{2 \gamma}} \frac{L_{j}}{P_{j}^{2}} \approx \frac{L_{j}}{M_{j}}$

One arrives practically at the same result if one uses the so called Hardy-Littlewood "conjecture B" [6]. This suggests that our approach is on the right track. Therefore:

Proposition no. 29: The number of twin ranks in a remnant from a super-group is on the order of the ratio of the length of the super-group to the length of its first interval. The fact that the number of twin ranks in an interval is proportional to the length of the interval is not surprising. What is really intriguing is the role played by the front twin ranks in determining the total number of twin ranks in a remnant, because eq. (7) implies $L_{T j} \approx R_{j} / F_{T j}$.

\section{CONCLUDING REMARKS}

This paper does not pretend to have solved the Twin Prime Conjecture. Its main objective was to show that despite the apparent randomness associated with twin primes (and hence with their correspondents twin ranks) there is an order among their complements in the set of positive integers, the non-ranks. These numbers form infinite series of consecutive groups and super-groups with an inner symmetry, a precise interval length and a well-defined number of terms. The fact that the non-ranks have a deterministic character makes their properties much easier to prove than in the case of twin primes. For example, (based on what was shown above) a rigorous proof of the Propositions 10 and 19 would make the infinity of twin prime almost certain. 
As shown, a minimum requirement for having only nonranks in a remnant (except for the front twin ranks) is that all terms in the second interval be covered by a single prime. But a single prime can cover only a fraction of the terms in the second interval; hence the uncovered terms must be twin ranks. To these twin ranks one still has to add the twin ranks in the following intervals $M_{j+2} . . M_{j+3}, M_{j+3} . . M_{j+4}$, $M_{j+4} . . M_{j+5}$ etc. inside the same remnant, that were not covered by the corresponding primes for the same reason: too many numbers, too few primes. Paradoxically, it is the paucity of primes that allows for the existence of twin primes.

\section{CONFLICT OF INTEREST}

None declared.

\section{ACKNOWLEDGEMENT}

None declared.

\section{REFERENCES}

[1] Guy RK. Unsolved problems in number theory. New York: Springer-Verlag 2004.

[2] Hardy GH, Wright EM. An Introduction to the Theory of Numbers. Oxford: UK 1979.

[3] Hardy GH, Littlewood JE. Contributions to the theory of the Riemann zeta-function and the theory of the distribution of primes. Acta Math 1916; 41: 119-6.

[4] Wrench, JW, Evaluation of Artin's constant and the twin prime. Constant Math Comput 1961; 15: 396-8.

[5] Havil, J. Gamma: Exploring Euler's Constant. USA: Princeton University Press 2003.

[6] Hardy GH, Littlewood JE. On some problems of "partitio numerorum." III: On the expression of a number as a sum of primes. Acta Math 1923; 44: 1-70.

Received: January 22, 2012

Revised: March 25, 2012

Accepted: April 2, 2012

(C) A. Dinculescu; Licensee Bentham Open.

This is an open access article licensed under the terms of the Creative Commons Attribution Non-Commercial License (http://creativecommons.org/licenses/by$\mathrm{nc} / 3.0 /$ ), which permits unrestricted, non-commercial use, distribution and reproduction in any medium, provided the work is properly cited. 